Case report

\title{
Primary extradural ectopic orbital Meningioma.
}

Zgolli Hsouna Mehdi 1,3, ${ }^{*}$, Mabrouk Sonya 1,3, Yeadeas Dahmeni 2,3, Zeghal Imen ${ }^{1,3}$, Fekih Olfa1, ${ }^{1,3}$, Yeadeas Monther ${ }^{2,3}$.

1: Department of Ophthalmology, Hedi Raies Institute, Tunis, Tunisia 2: Department of ophthalmology, Military Hospital, Tunis, Tunisia 3: College of medicine, Tunis, Tunisia * Corresponding author Correspondence to: hsouna_zgolli@yahoo.com Publication Data: Submitted: January 02,2021 Accepted: April 12,2021 Online: May 30,2021

This article was subject to full peer-review.

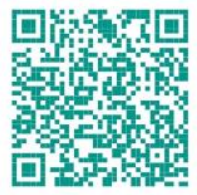

This is an open access article distributed under the terms of the Creative Commons Attribution Non-Commercial License 4.0 (CCBY-NC) allowing sharing and adapting. Share: copy and redistribute the material in any medium or format. Adapt: remix, transform, and build upon the licensed material.

The work provided must be properly cited and cannot be used for commercial purpose.

\section{Abstract}

Orbital meningioma is a rare benign tumor. However, the existence of ectopic orbital meningiomas was debated for long time and this lesion might be underreported. Complete surgical excision could achieve a good prognosis without visual impairment. The recurrence rate is still considerable. The aim of this report was to highlight the diagnostic difficulties and management characteristics.

\section{Keywords}

Orbit; meningioma; origin, diagnosis; management.

\section{Introduction}

Orbital meningioma is a rare tumor. This entity is more frequently diagnosed in adult patients. Meningiomas that arise from the arachnoid layers of the optic nerve are considered as primary. Secondary orbital meningioma is defined as an extension of an intracranial process, usually from the sphenoidal wind [1]. Orbital meningioma may arise apart from optic nerve and without any connection with the intracranial meninges. In such cases the lesion is classified as ectopic orbital meningioma [2]. This entity is very rare; few cases are reported in the literature. Its origin is still controversial. We herein, report a case of an ectopic orbital meningioma with compressive optic neuropathy.

\section{Observation}

We report the case of a 37-year-old female presenting high myopia (-6.25 in both eyes). She complained three months after childbirth of progressive protrusion of the right eye, decreased visual acuity and amputation of the lower visual field. A swelling of the upper eyelid appeared later. The examination of the left eye was normal. The examination of the right eye diagnosed an axial and a non-reducible exophthalmos and a best corrected visual acuity of only $2 / 10$. Eye fundus showed papillary edema stage 2 . Otherwise, the physical examination was normal.

The Lancaster's test was normal. The Goldman visual field test revealed enlargement of the of the blind spot. Orbital tomography showed a right exophthalmos grade 2, with a right retro-orbital mass of $2 \mathrm{~cm}$. The lesion had cystic and tissular mixed pattern and partially enhanced after contrast injection. MRI scan right intra-conical and retro orbital mass of 22x20 with isoT1 and hyper T2 signals and heterogeneous enhancement after gadolinium injection. A mass effect was noted on the right eye and optic nerve without invasion (figure1).

Surgery was indicated and performed by multidisciplinary team including neurosurgeon and ophthalmologist. A right sub frontal approach with orbital roof section was indicated (figure2). The tumor was completely resected, and the specimen was sent to the pathology department. Histological examination concluded to transitional meningioma grade1. The postoperative course was marked by ophthalmoplegia and ptosis which regressed completely after 3 months. The ophthalmological examination at 1 month showed visual acuity of $6 / 10$ and mild papillary edema. Increase of the visual acuity to $8 / 10$ and normal fundus examination were seen later at 6 months. No recurrence of the tumor was noted in The MRI scan done 2 years after the procedure. 

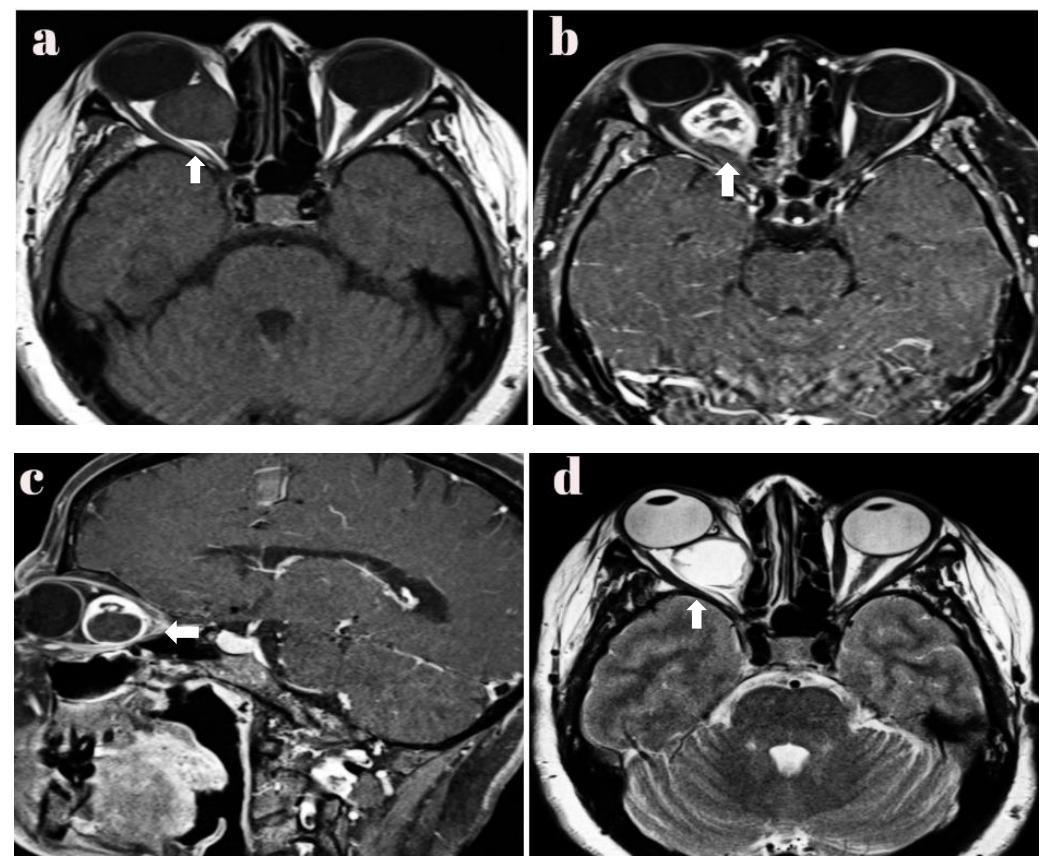

Figure 1: Orbito-cerebral MRI : (a) T1 axial image: well limited mixed intra right retro-orbital tissular mass $22 \times 20 \mathrm{~mm}$; iso intense T1. $(b, c)$ : heterogeneous enhancement after gadolinium injection. (d): T2 axial image: hyperintense tissular mass signal.
We noted the independence of the mass which is only compressing the optic nerve.
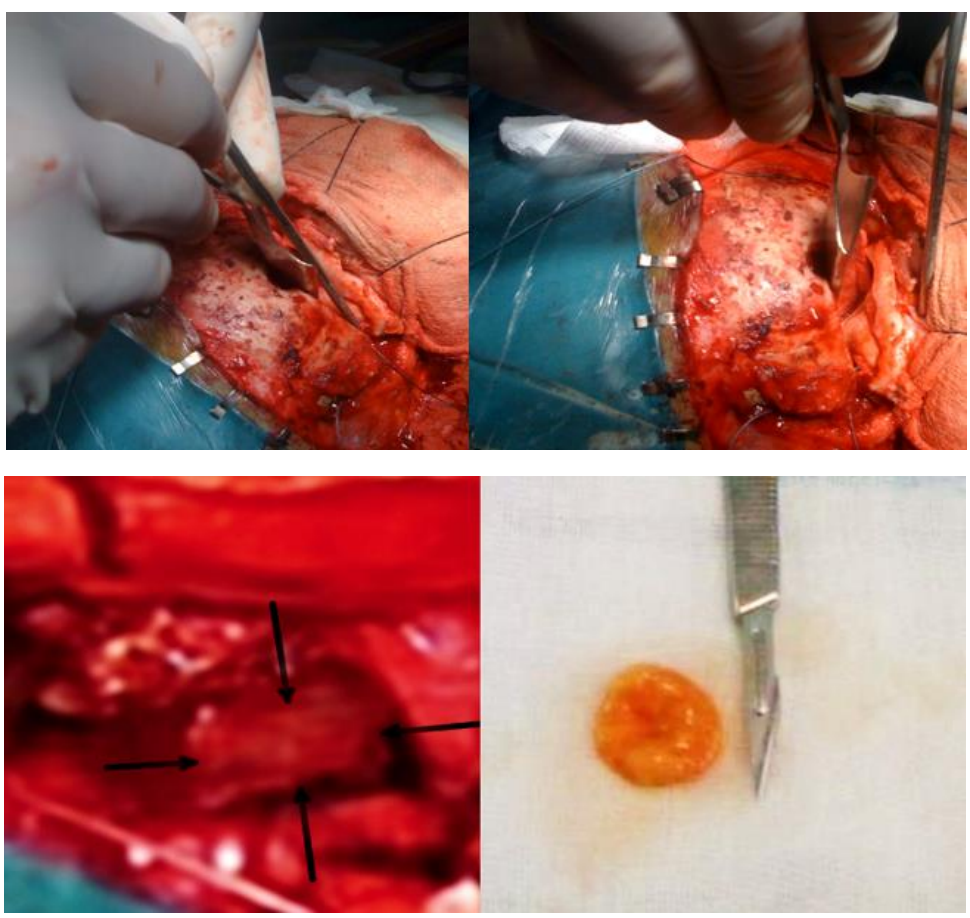

Figure 2: Surgical site access and resected tumor features. 
Discussion

Meningiomas are one of the most frequent neoplasms arising from the meninges. These tumors belong to the arachnoid villi structures [3]. Commonly, meningiomas are attached to the dura and grow in the cranial cavity or intraspinal region. However, several ectopic location such as mandibular or parapharyngeal have been described. The orbital ectopic meningioma remained controversial, and less than 25 cases were reported till 2018 [4]. Primary orbital meningioma accounted for $5-10 \%$ of all orbital neoplasms and mainly diagnosed in adult patients Primary orbital meningiomas originate from the arachnoid layer of the optic nerve sheath. Approximately $70 \%$ of orbital meningiomas are intracranial neoplasms originating at the sphenoid ridge, with orbital and intraluminal extensions [5]. The debate about its origin evidence may be related to the paucity or incomplete character of the of the published series. Congenital and traumatic causes have been evoked by some authors[6]. The preoperative diagnosis is difficult because of the non-specificity of the symptoms. The magnetic resonance scan may contribute to distinguish this tumor from the other neoplasms of the sphenoid wing and remain the standard for diagnostic and follow up imaging [7]. Surgical excision is the gold standard of the management and must be complete to avoid recurrence. The management of recurrent tumors is more challenging and may require external beam radiotherapy (EBRT) with all its possible retinal side effects [8]. In this report we tried to demonstrate that orbital meningioma may arise apart from optic nerve and without any connection with the intracranial meninges. This could make reasonable to consider it as ectopic despite the confusing anatomical factors.

\section{Conflict of Interest: None}

\section{References}

[1] Satija CE, Mehta V], Maltry AC, Nussbaum E, Nussbaum L, Harrison AR. A report of intracranial meningioma recurring as ectopic orbital meningioma associated with basal encephalocele. Ophthalmic Plast Reconstr Surg. 2020;36:e19-e21.

[2] Tendler I, Belinsky I, Abramson DH, Marr BP. Primary extradural ectopic orbital meningioma. Ophthalmic Plast Reconstr Surg. 2017;33:S99-S101.

[3] Tan LT, Stewart CM, Sheerin F, Mac Donald B, Silva P, Norris JH. Ectopic orbital meningioma: Fact or fiction? Orbit. 2017;36:144-46.

[4] Yeşiltaş YS, Gündüz K, Okçu Heper A, Erden E. Ectopic rhabdoid meningioma of the orbit in a child: case report and review of the literature. J Neurosurg Pediatr. 2018;22:151-57.

[5] Huang X, Tang D, Wu T, Jian T, Sun F. Ectopic orbital meningioma: a retrospective case series. BMC Ophthalmol. 2018;18:296.

[6] Gündüz K, Kurt RA, Erden E. Ectopic orbital meningioma: report of two cases and literature review. Surv Ophthalmol. 2014;59:643-8.

[7] Lee DH, Sim HS, Hwang JH, Kim KS, Lee SY. Extracranial Meningioma Presenting as an Eyebrow Mass. J Craniofac Surg. 2017;28:e305-e307.

[8] Luzzi S, Zoia C, Rampini AD, Elia A, Del Maestro M, Carnevale S,et al. Lateral transorbital neuroendoscopic approach for intraconal meningioma of the orbital apex: Technical nuances and literature review. World Neurosurg. 2019;131:10-17. 